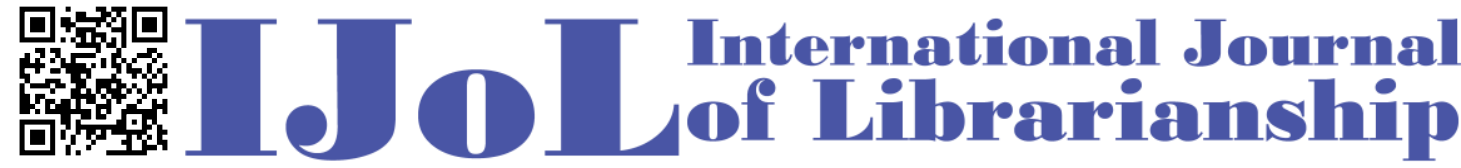

ISSN: 2474-3542 Journal homepage: http://journal.calaijol.org

\section{Usage Analysis of University of Manitoba Licensed Databases via EZproxy's Logs}

\section{Wei Xuan}

\begin{abstract}
:
Compared to vendor-provided data, Transaction Log Analysis (TLA) can provide unique benefits to a library in analyzing database usage. Meanwhile, it also requires librarians to have a broad knowledge of Information Technology in order to implement a TLA. This article will present an in-house database system developed at the University of Manitoba Libraries to analyze EZProxy's Starting Point Uniform Resource Locator logs. The database visits that happened in the past two years have been imported into this system and are split into two categories, on-campus and off-campus visits. Unique statistical information from each category is also discussed.
\end{abstract}

To cite this article:

Xuan, W. (2019). Usage analysis of University of Manitoba licensed databases via EZproxy's logs. International Journal of Librarianship, 4(2), 76-93.

To submit your article to this journal:

Go to http://ojs.calaijol.org/index.php/ijol/about/submissions 


\title{
Usage Analysis of University of Manitoba Licensed Databases via EZproxy's Logs
}

\author{
Wei Xuan \\ University of Manitoba Libraries, Winnipeg, Manitoba, Canada
}

\begin{abstract}
Compared to vendor-provided data, Transaction Log Analysis (TLA) can provide unique benefits to a library in analyzing database usage. Meanwhile, it also requires librarians to have a broad knowledge of Information Technology in order to implement a TLA. This article will present an in-house database system developed at the University of Manitoba Libraries to analyze EZProxy's Starting Point Uniform Resource Locator logs. The database visits that happened in the past two years have been imported into this system and are split into two categories, on-campus and offcampus visits. Unique statistical information from each category is also discussed.
\end{abstract}

Keywords: Database Usage Analysis, Transaction Log Analysis (TLA), EZProxy, SPULog

\section{INTRODUCTION}

A database is "a structured set of data held in computer storage and typically accessed or manipulated by means of specialized software"(OED, 2019). To academic libraries, the term database represents searchable online resources. A database may provide users access to abstracts, or full-text articles, or images, etc. The access is usually paid by the library. Since the start of migrating content from print materials to online databases, libraries have been looking for methods to better analyze the usage of licensed e-resources. The analysis of database usages should allow librarians to know how different databases are used by different groups of users and what the user groups preferred. Discovery tool and information sources are most likely chosen when users perform information searches. With the knowledge of such information-seeking behaviors, librarians will be able to provide custom services to particular user groups. On the other hand, analyzing database usages also allows libraries to better estimate the value of a particular database to their institutions, such as whether or not it is a good investment to subscribe to a database. Especially with more universities moving to a faculty-oriented budget model and when budget cuts happen more frequently, the need to demonstrate how licensed databases are used by faculties is becoming more urgent in the decision-making process.

This article analyzed the usage of licensed databases at University of Manitoba for a period of two years (September $1^{\text {st }}, 2016$ - August 31 ${ }^{\text {st }}, 2018$ ). A typical EZProxy Uniform Resrouce Locator (URL) is like https://abclib.idm.oclc.org/login?url=https://www.jstor.com . The first part of the URL is the proxy prefix (https://abclib.idm.oclc.org/login?url=), which is the EZProxy 
server's URL, and the rest is the target URL (https://www.jstor.com ), which is the unique database URL. The proxy prefix and the target URL make a starting point URL(SPU) (OCLC, 2018b). The proxy prefix stays the same for all resources licensed by an institution. The target URL represents the specific e-resource that a user visited. This database usage analysis is based on the EZProxy SPU logs as the target URLs are recorded each time a starting point URL is clicked (OCLC, 2019). Compared to normal EZProxy logs, SPU logs greatly simplify the analysis process and allow librarians to focus on analyzing how the user accesses a particular database URL without being distracted by information, such as text, images, etc., transferred between the database website and the EZproxy server.

The author designed a MySQL database and imported EZProxy's SPU logs into it. This database also contains the University of Manitoba (UofM) campus Internet Protocol (IP) ranges, UofM Identity (ID) for faculty, staff and students, and all database records from the Libraries' AZ Database list on LibGuide. With this database, the author was able to identify the following:

1. Where was a database visit made? Was it from campus or off-campus?

2. How a database visit was initiated? Did the user start from the Libraries' AZ Database List or the discovery platform - Primo?

3. How frequently was a database visited during a specific period? Regarding on-campus visits, how many visits were from the wireless network and how many from Ethernet in a particular building, such as the library building? Regarding off-campus visits, how many visits were made from a particular user group (faculty, graduate students, and undergraduate students)?

4. What was the preference for a particular user group when they visited databases off-campus? For example, what are the top 10 databases used by graduate students when they are offcampus?

Thus, the database analysis method described in this article allows the author to investigate both the usage of a particular database in the period and the off-campus information-seeking activities of a particular user group in the time frame.

\section{LITERATURE REVIEW}

There are generally two major methods for librarians to know how their subscribed databases are used among users. One is through vendor-supplied usage data and the other is through transaction log analysis (TLA)(Peters, 1993). Each method has its advantages and disadvantages (Duy \& Vaughan, 2003).

With the vendor-supplied usage data, the issues can be generalized as a lack of credible and comparable data. During the early implementation, different vendors were using various types of measures in their reports. Due to the lack of context for the data, such as how the number was counted and collected, librarians had great difficulty in comparing and interpreting the usage data (Blecic, Fiscella, \& Wiberley, 2001). To solve the problem, an international organization, COUNTER (Counting Online Usage of Networked Electronic Resources), was formed to promote a consistent way for e-resource providers to generate usage reports (COUNTER, 2019). By releasing the Code of Practice, COUNTER is promoting standards in this area. The consistency of 
usage data from the vendors that are COUNTER-compliant has been improved. However, even COUNTER-compliant vendors still might be able to count searches differently without violating the Code of Practice (Blecic, Fiscella, \& Wiberley, 2007). Besides, since it is not mandatory, some vendors haven't applied the COUNTER Code of Practice.

The challenge for librarians with the COUNTER reports is that it is very difficult and timeconsuming to put together reports from all vendors. To a research library that usually subscribes to hundreds of databases, it becomes an impossible mission for their librarians to group all the COUNTER data by themselves. To provide a solution, the National Information Standards Organization (NISO) initiated a working group to work on Standardized Usage Statistics Harvesting Initiative (SUSHI) in 2005 and approved the SUSHI standard in 2007 (NISO, 2019). It automates the transfer of usage data from vendors to a central system, whether it is the Integrated Library System (ILS) or a reporting portal.

Even with COUNTER and SUSHI, there are still disadvantages to solely rely on vendorprovided usage data. The biggest issue is that the vendor cannot have the user's information. The most commonly used authentication method for a database visit request is still the IP authentication. Approving a request simply based on whether it's from a registered IP address, the database provider is unable to include user information in the statistics. As a result, a database vendor can provide the number of searches but cannot answer questions like who made those searches. Even with the Security Assertion Markup Language (SAML) authentication, such as Shibboleth (Shibboleth, 2019), the user's attribute released to the database provider may only contain basic group information, such as faculty and student. Secondly, as library consortium becomes the trend in managing resources and negotiating licenses (Liu \& Fu, 2018), member libraries in a consortium may lose statistical information about their usage. This issue has been existing in the public library environment for a long time. Thirdly, it is mentioned by Wan and Liu (Wan \& Liu, 2010) that a library may have more than one account with a database provider.

Despite the inconveniences in utilizing vendor-supplied usage data, a survey carried out in 2006 (Baker \& Read, 2008) found that the majority of research libraries relied on it to make subscription decisions, justify expenditures and meet reporting requirements.

TLA is to analyze the transaction logs of a proxy server that users have to use to access the licensed e-resources. If an e-resource visit can bypass the proxy server, such as campus IP addresses directly recognized by the database provider or SAML authentication on the database provider's website, that visit will not be recorded in the proxy server's log. Thus, the library will lose certain statistical information about those kinds of visits when performing TLA. The advantage of TLA is that librarians have first-hand access to raw data. If well analyzed, those logs will provide a better demonstration of user activities. However, the difficulty with TLA is how to retrieve useful information from the log, as a web server log usually contains all kinds of web activities and is saved as a text file, and how to group log files, as log files are usually created daily. Those challenges require librarians to either have a strong background in Information Technology (IT) or work closely with IT staff in order to understand and analyze transaction logs.

Coombs conducted a project at the State University of New York College at Cortland (SUNY Cortland), which analyzed EZProxy transaction logs (Coombs, 2005). EZProxy is the 
commercial product that SUNY Cortland used to access databases. This project demonstrated that librarians could use TLA to study user information behavior on accessing the library's e-resources. Unfortunately, the author didn't demonstrate whether their method was able to track how the database visits change with time. The author also didn't give many details about how their system used to do TLA was designed and developed and how EZProxy log files were imported into this system. Without such information, it would be very hard for other institutions to reproduce the work.

Gonzales published a way to automatically parse EZProxy's SPU log (Gonzales, 2018). Since OCLC doesn't provide any tools to analyze EZProxy transaction logs and the commercial tools on the market are not optimized for EZProxy, Gonzales developed a Python script to import raw data from EZProxy SPU logs into a single CSV file and used two additional Python modules, Pandas and Matplotlib, to analyze it. The issue with this method is that the analysis of data stored in a CSV file is limited by the function of these two Python modules. Considering the number of records stored in the CSV file, it would cause a great load for a workstation to open it, not to mention to run any possible queries to it.

In this article, the author presented the database developed at the University of Manitoba Libraries to analyze EZProxy SPU logs. EZProxy SPU logs generated after September 1 ${ }^{\text {st }}, 2016$, have been being imported. The author analyzed all the visits to licensed e-resources within a period of two years (Sept. $1^{\text {st }} 2016$ - August 31 ${ }^{\text {st }}$, 2018). In this article, "database platform" means the eresource provider's website where users can search articles, images, etc. Several databases may share one database platform. For example, EBSCO has many databases, such as Academic Search Complete, Ageline, Alternative Press Index, etc. All the databases are using the same database platform, search.ebscohost.com. Alexander Street Press also hosts several databases. However, the websites for the databases are different. For example, the website for the American Film Scripts Online database is http://solomon.afso.alexanderstreet.com/ and the website for the Asian American Drama database is http://solomon.aadr.alexanderstreet.com. They will be treated as different database platforms in the analysis.

If a user goes to the Libraries' AZ database list on LibGuide and clicks the link to visit a specific database, in this article, the visit is treated as from the AZ database list. If a user uses the library's discovery platform, Primo from Ex Libris, to find an article and clicks the link to access it on the database platform, the visit is treated as from Primo.

\section{METHODOLOGY}

At the University of Manitoba Libraries, the licensed databases are set up to only allow visits from the EZProxy server. Thus, the EZProxy log records all the starting point URLs in the visits to our licensed e-resources, no matter whether the visit is from campus or off-campus.

When receiving a database-visit request, EZProxy will first check the requester's IP address. UofM IP ranges are whitelisted by the EXProxy server. If the request is from an UofM IP address, which means the user is on campus, EZProxy will automatically grant access to the user. In the log file, the word "auto" is recorded as the user identity (ID) for this visit. If the request is not from a UofM IP address, which means the user is off-campus, EZProxy will direct the user to a login 
page where she/he needs to use the library ID to authenticate. For UofM faculty, staff and students, EZProxy uses SAML 2.0 protocol to authenticate them against signUM which is a central identity system used at UofM. In EZProxy log, the user's UMnetID, which is the user's unique identifier in signUM, will be recorded as the username for this visit. In both cases, the user's IP address will be stored in the EZProxy log file.

We are using the following value for the EZProxy LogSPU(OCLC, 2018a) directive.

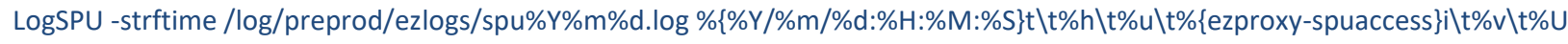
-strftime is to record starting point URL information in a new log file each day

$\% \mathrm{t}$ is the Date/time of request;

\%h is the IP address of the host accessing EZProxy

$\% u$ is Username used to log into EZproxy

$\% v$ is the hostname of the webserver

$\% \mathrm{U}$ is the requested URL

A typical record in EZProxy log is like

2016/09/07:15:54:01 140.193 .167 .125 auto proxy www.jstor.org
http://www.jstor.org/action/showPublication?sid=primo\&journalCode=science

A script has been developed to automatically import EZProxy SPU logs into a table, called "EZProxySPULog", in a MySQL database, which also contains information about user groups, IP addresses on campus and A-Z database list that is used in our subject guide. The EntityRelationship Diagram is presented in Figure 1.

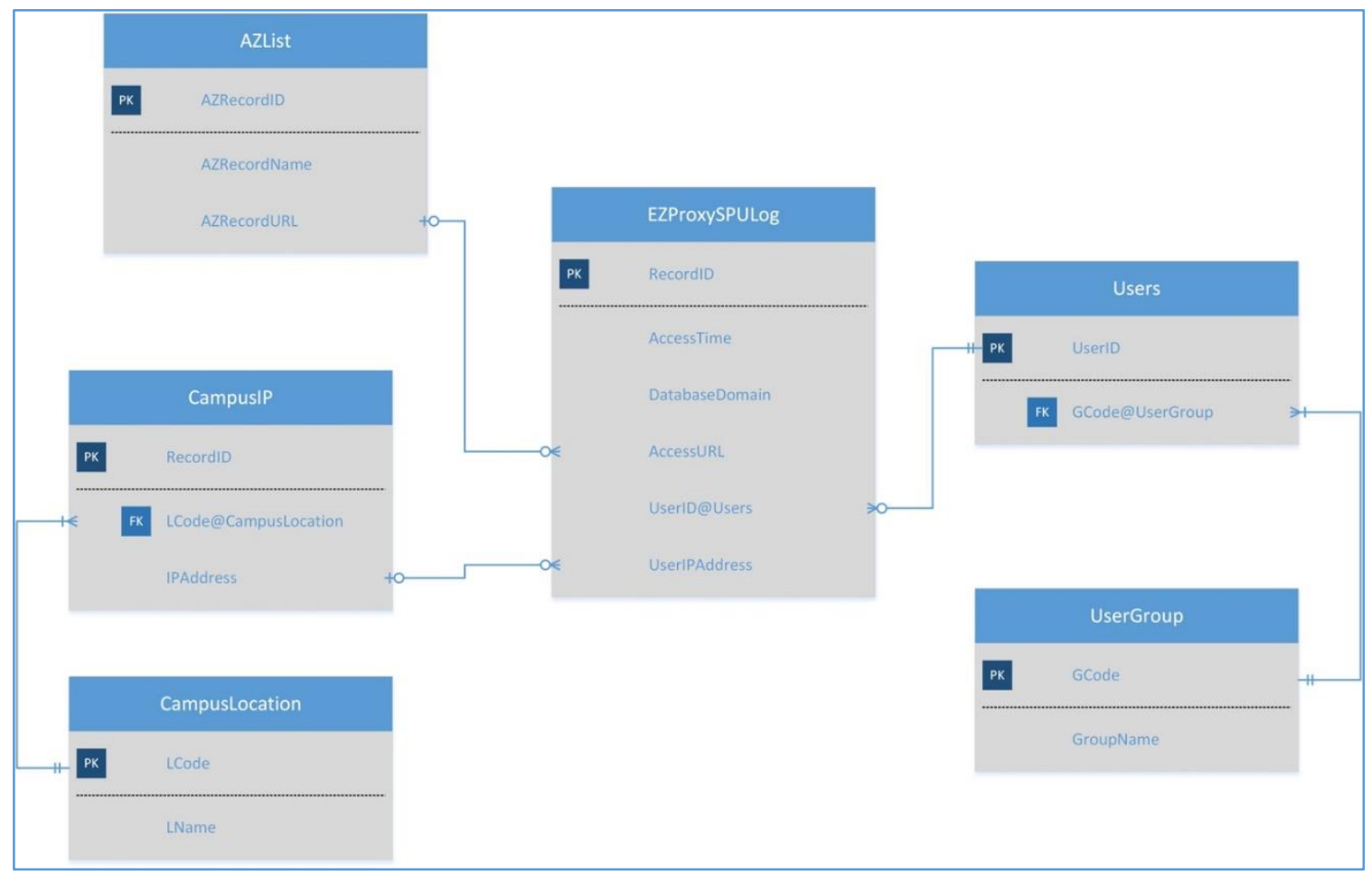

Figure 1. Entity Relationship Diagram for the database 
The EZProxyLog table splits each visit record in the EZProxy log into five different fields, Access Time, Database Domain, Access URL, User ID, and User IP Address. The above log record is stored in the EZProxySPULog table in the following way (Table 1).

Table 1. Sample of a record in the EZProxySPULog table

\begin{tabular}{|c|c|c|l|c|c|}
\hline id & datetime & host & \multicolumn{1}{c|}{ URL } & user_id & IP \\
\hline 201609073592 & $2016-09-07$ 15:54 & www.jstor.org & $\begin{array}{l}\text { http://www.jstor.org/action/showPublication?sid=p } \\
\text { rimo\&journalCode=science }\end{array}$ & auto & 140.193 .167 .125 \\
\hline
\end{tabular}

The SQL query to retrieve the amount of on-campus/off-campus/total database accesses is

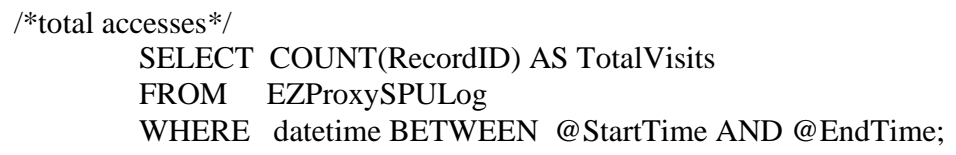

The Libraries Systems department at the University of Manitoba Libraries gets a list of all UofM students from the IT department each semester. The list contains student name, UMnetID, the affiliated faculty and contact information. Graduate students are affiliated with Faculty of Graduate Studies on the list. Undergraduate students are affiliated with their respective faculty, such as Faculty of Science, Faculty of Engineering, etc. Data from this list is desensitized and only UMnetID and the affiliated faculty are used in the following two tables, Users and Usergroup, in the database. Since each student's UMnetID is unique, it is used for the UserID field, which is the primary key in the table "Users". There are 29 major academic units (faculty, college, school, etc.) that a student may be associated with at the university. These 29 units are stored in the UserGroup table. Each unit is a group in this database and assigned a 3-digit number as an identifier. Regarding other users, such as faculty, staff, alumni, retirees, etc., since they are in respective user groups in our Integrated Library System (ILS), we use their group information from ILS in this database. Groups 1-15 and 17-29 are used for undergraduate students. Group 16 is for graduate students. All UofM faculty and staff, including visiting scholars, are in a different group (GCode:100). Unfortunately, we cannot know the rank (Professor, Associate Professor, Assistant Professor, etc.) of a specific user in Group 100 as such information is not used in the ILS. We also don't know which faculty a specific user in Group 100 is affiliated with. The UserGroup table is attached in Appendix I. Alumni have access to a small portion of our licensed databases. Local community users have no remote access to our e-resources. Thus, no specific user groups were created in the UserGroup table for these two user categories. Since UserID is recorded in the SPU log for offcampus accesses (Table 2), we can combine three tables, EZProxySPULog, Users and UserGroup, 
in the database, to analyze which user group made what kind of database access.-Some SQL queries are presented in the following.

Table 2. Sample of an off-campus visit in the EZProxySPULog table

\begin{tabular}{|c|c|c|l|l|c|}
\hline id & datetime & \multicolumn{1}{|c|}{ host } & \multicolumn{1}{c|}{ URL } & user_id & IP \\
\hline 2016082022 & $2016-08-200: 18$ & www.jamaevidence.com & $\begin{array}{l}\text { http://www.jamaevidence.com/resource/ } \\
523\end{array}$ & A $^{* * * * *}$ & $2^{* * * * . *}$ \\
\hline
\end{tabular}

Note: user_id and IP are masked by the author.

$/ *$ number of accesses filtered by group*/

SELECT UserGroup.GName AS UserGroup, COUNT (EZProxySPULog.RecordID) AS NumberOfVisits

FROM EZProxySPULog INNER JOIN Users

ON_EZProxySPULog.UserID@Users= Users.UserID

INNER JOIN UserGroup

ON Users.GCode@UserGroup=UserGroup.GCode

WHERE datetime BETWEEN @StartTime AND @EndTime

GROUP BY UserGroup

ORDER BY NumberOfVisits DESC;

A database A-Z list is presented to users on the Libraries' website. Database names and domains from this list are also inputted into the AZList table in this database. If a record in the AccessURL field in the EZProxyLog table matches a record in the AZRecordURL field in the AZList table, this visit is regarded as being initiated from the A-Z list on the Libraries' website as the user has to either click the database link on the A-Z list or use a bookmarked URL which is originally from the A-Z list.

UML is using Ex Libris' product, Primo, as the discovery platform. The article's hyperlink on Primo will direct EZProxy to proxy the article's URL from the database platform. A typical request EZProxy receives from Primo is like the following URL:

https://uml.idm.oclc.org/login?\&url=http://www.jstor.org/openurl? sid=primo\&volume=335\&au last $=$ Ponomarenko\&date $=2012 \&$ spage $=947 \&$ issn $=00368075 \&$ issue $=6071$

In this case, the AccessURL field for this access will be the article's full URL

http://www.jstor.org/openurl? sid=primo\&volume $=335 \&$ aulast $=$ Ponomarenko\&date $=2012 \&$ spa $g e=947 \& i s s n=00368075 \&$ issue $=6071$ instead of the database's URL http://www.jstor.org. Thus, if a value in the AccessURL field in the EZProxySPULog table matches a value in the AZRecord field in the AZList table, the access is from the AZ database list. Otherwise, access is from the library's discovery platform.

/*-------------Top 10 databases based on visit from AZ list----*/

SELECT AZList.AZRecordName AS DatabaseName, COUNT( EZProxySPULog.RecordID) AS NumberOfVisits

FROM EZProxySPULog LEFT JOIN AZList

ON EZProxySPULog.AccessURL= AZList.AZRecordURL

WHERE EZProxySPULog.AccessTime BETWEEN @starttime AND@endtime

AND AZList.AZRecordName IS NOT NULL

GROUP BY DatabaseName

ORDER BY NumberOfVisits DESC

LIMIT 10;

/*-------------Top 10 database platforms based on visit from Primo----*/

SELECT EZProxySPULog.host AS DatabasePlatform, COUNT( EZProxySPULog.id) AS NumberOfVisits

FROM EZProxySPULog LEFT JOIN AZList 
ON EZProxySPULog.AccessURL= AZList.AZRecordURL

WHERE EZProxySPULog.AccessTime BETWEEN@starttime AND@endtime

AND AZList.AZRecordName IS NULL

GROUP BY DatabasePlatform

ORDER BY NumberOfVisits DESC

LIMIT 10;

The University of Manitoba has its IP ranges. The IP ranges can be split into two major categories, Wi-fi and Ethernet. Regarding the Ethernet category, it can be further broken down into each building, such as the Library building, the Education building, etc. Regarding the Wi-fi category, a special range of IP addresses is reserved for graduate students, undergraduate students, and guest users. Considering the number of guest users is very small, most of the database visits from this special IP range are made from students. Thus, this IP-location information is stored in two tables in this database, CampusIP, and CampusLocation. For a record in the EZProxyLog table, if the value of the UserID@Users field is "auto", which means the visit is from a UofM IP address, we can check the location information. For example, if the IP address belongs to the Ethernet category, is it from the library building, the Science building or somewhere else? If the IP address belongs to the Wi-fi category, is it from the student and guest range? A sample query is presented in the following to group on-campus accesses based on location.

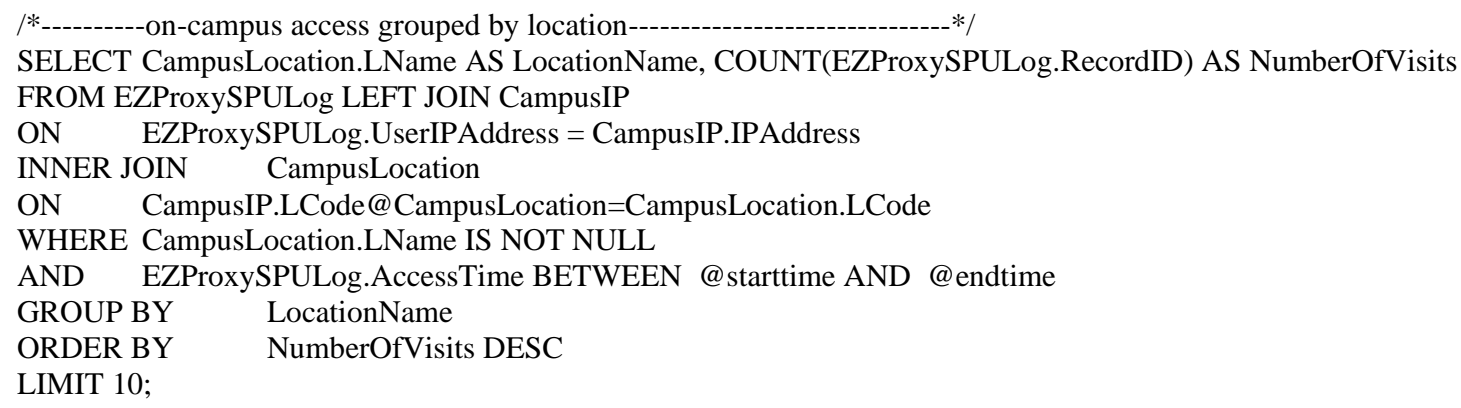

When combining all the tables in the database together, we will be able to study complicated usages such as:

1. How often is a specific database on the AZ list accessed by users located in a particular building on campus?

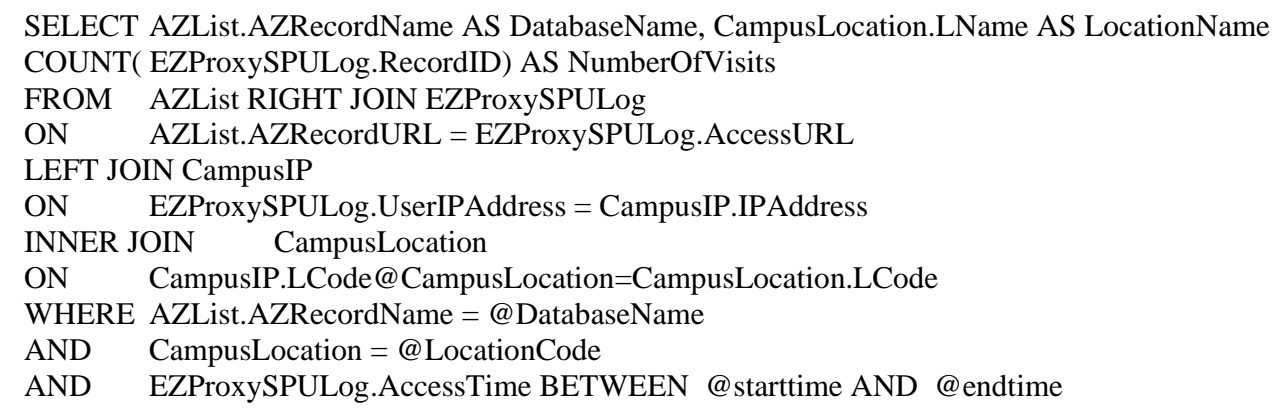

2. For users in a specific group, what database do they most frequently visit through the library's discovery platform when they are off campus?

SELECT EZProxySPULog.host AS DatabasePlatform, COUNT( EZProxySPULog.id) AS NumberOfVisits FROM AZList RIGHT JOIN EZProxySPULog ON AZList.AZRecordURL = EZProxySPULog.AccessURL INNER JOIN Users 


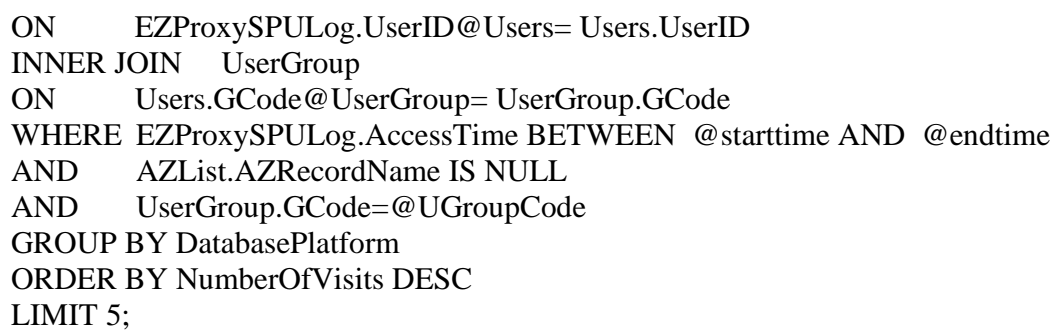

\section{RESULTS}

During the period analyzed in this report (September $1^{\text {st }}, 2016$ - August $31^{\text {st }}, 2018$ ), there were $5,061,398$ visits to our licensed databases. Categorized by user location, 1,865,251 visits were made on-campus while the rest are 3,196,147 visits from off-campus (Figure 2). How the on-campus and off-campus visits change with time can be found in Figure 3.



Figure 2. Database visits based on user location

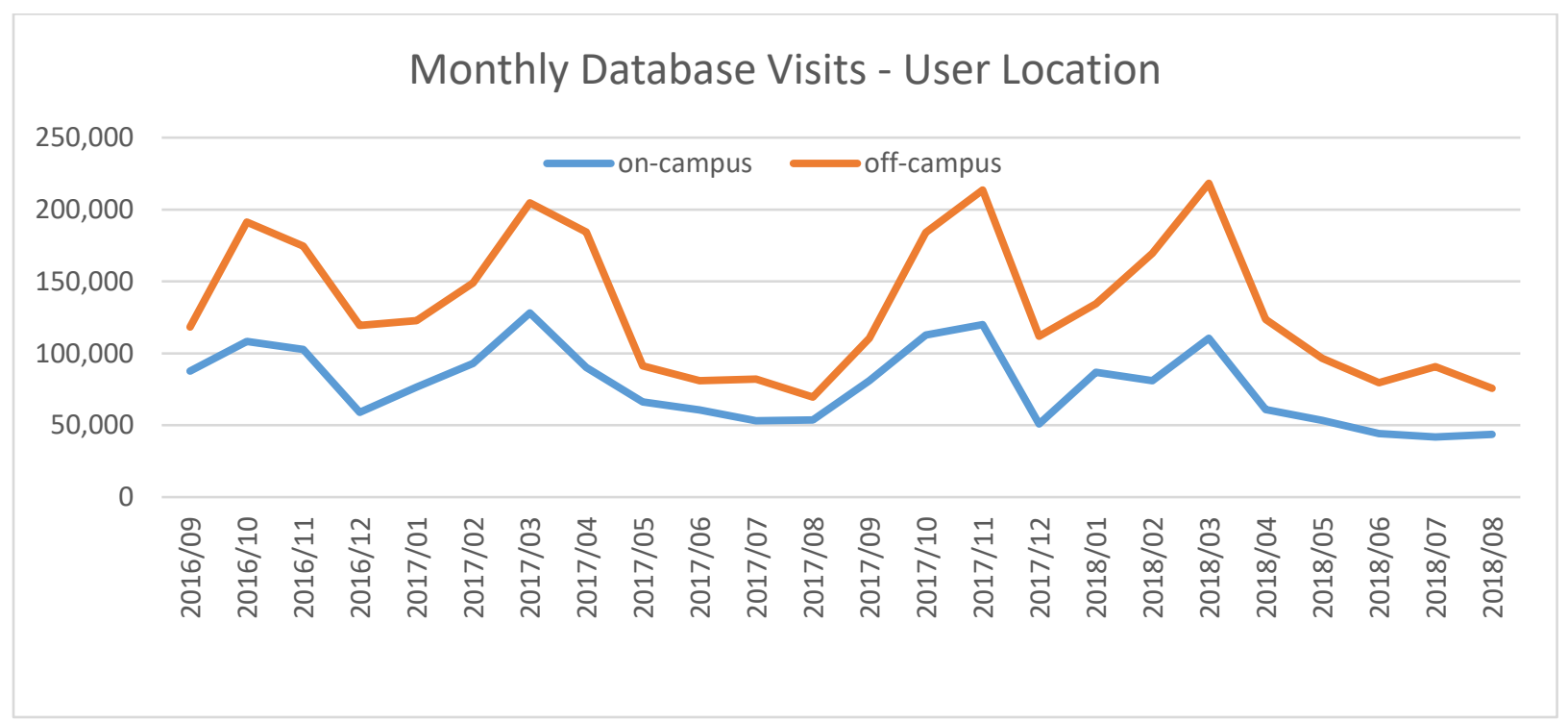

Figure 3. Monthly database visits - user location 
Categorized by discovery channel, 844,090 visits were made from the AZ database list; 4,217,308 visits were made from Primo (Figure 4). How many times each of the two channels were used in a specific month can be found in Figure 5.

\section{Database Visits - Discovery Channel}

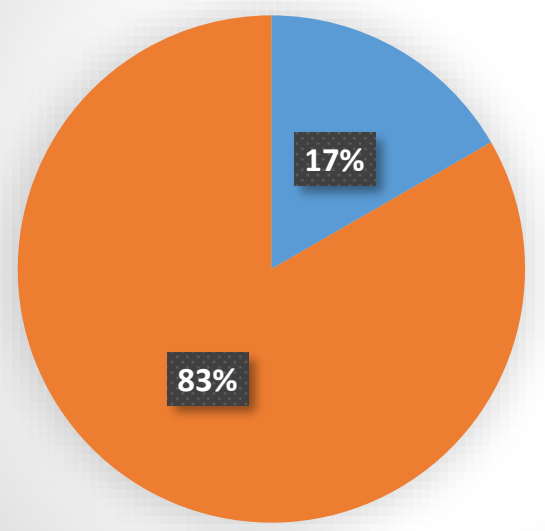

aZ Database List

- Primo

Figure 4. Database visit based on the discovery channel

\section{Monthly Database Visits - Discovery Channel}

- AZ list Primo

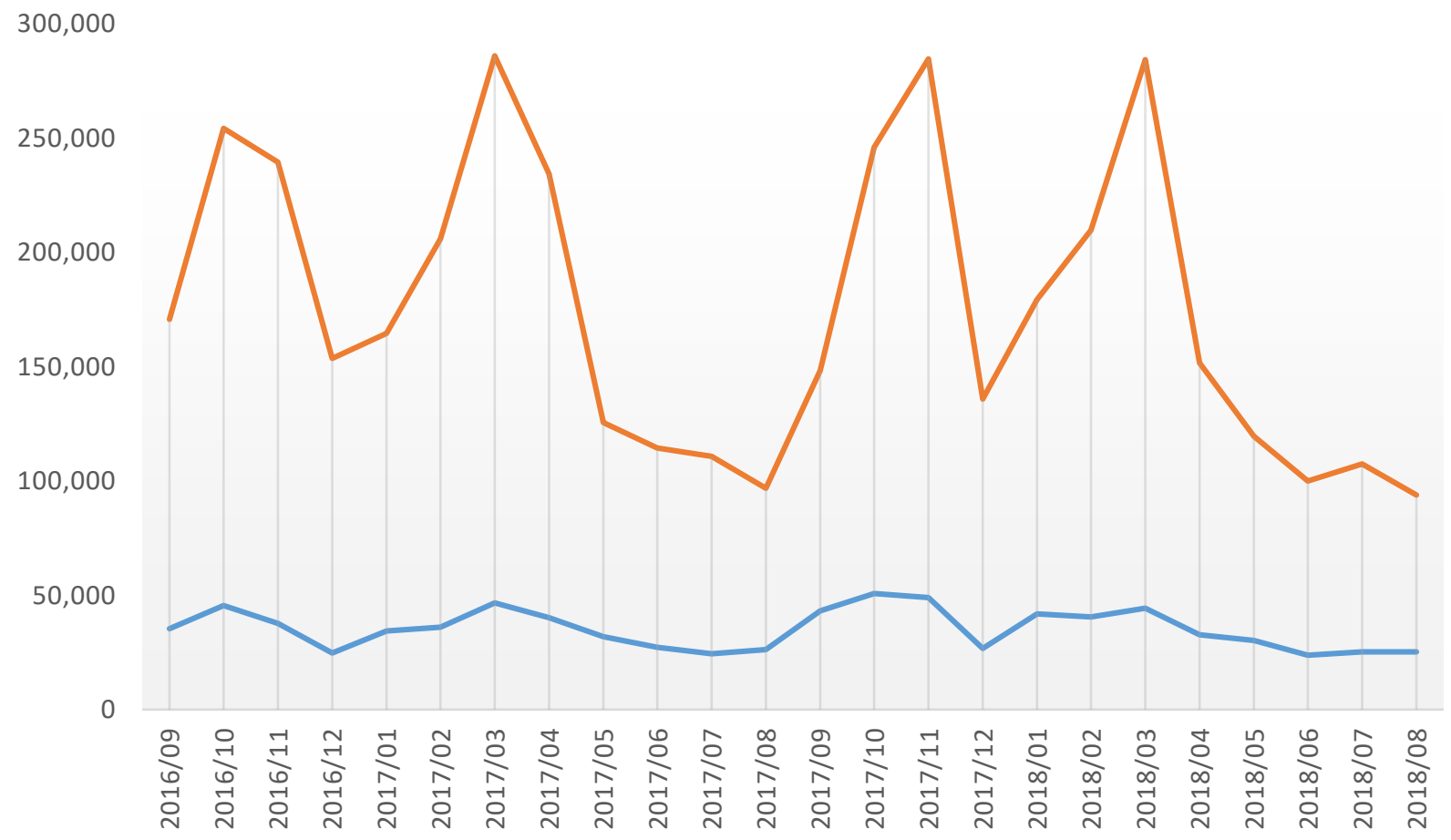

Figure 5. Monthly database visits - discovery channel 
Regarding the on-campus visits, 237,854 were made from the AZ database list while 1,627,397 were made from Primo (Figure 6). The monthly visits through these two channels can be found in Figure 7.

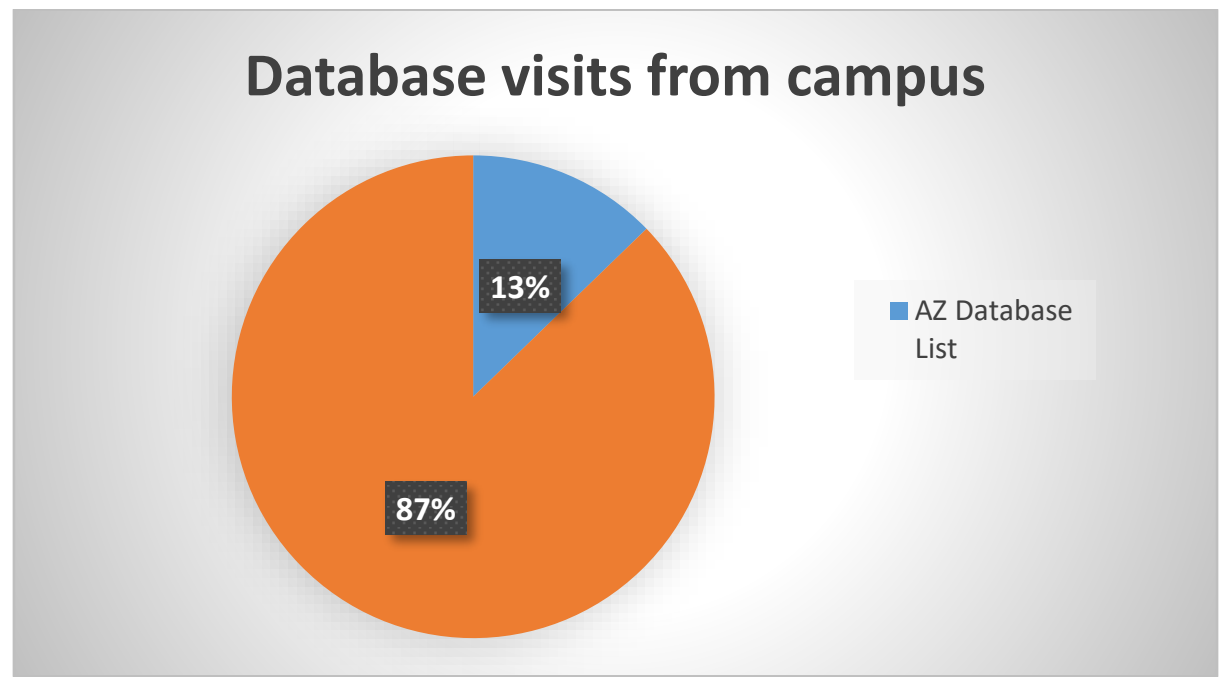

Figure 6. Database visits from campus

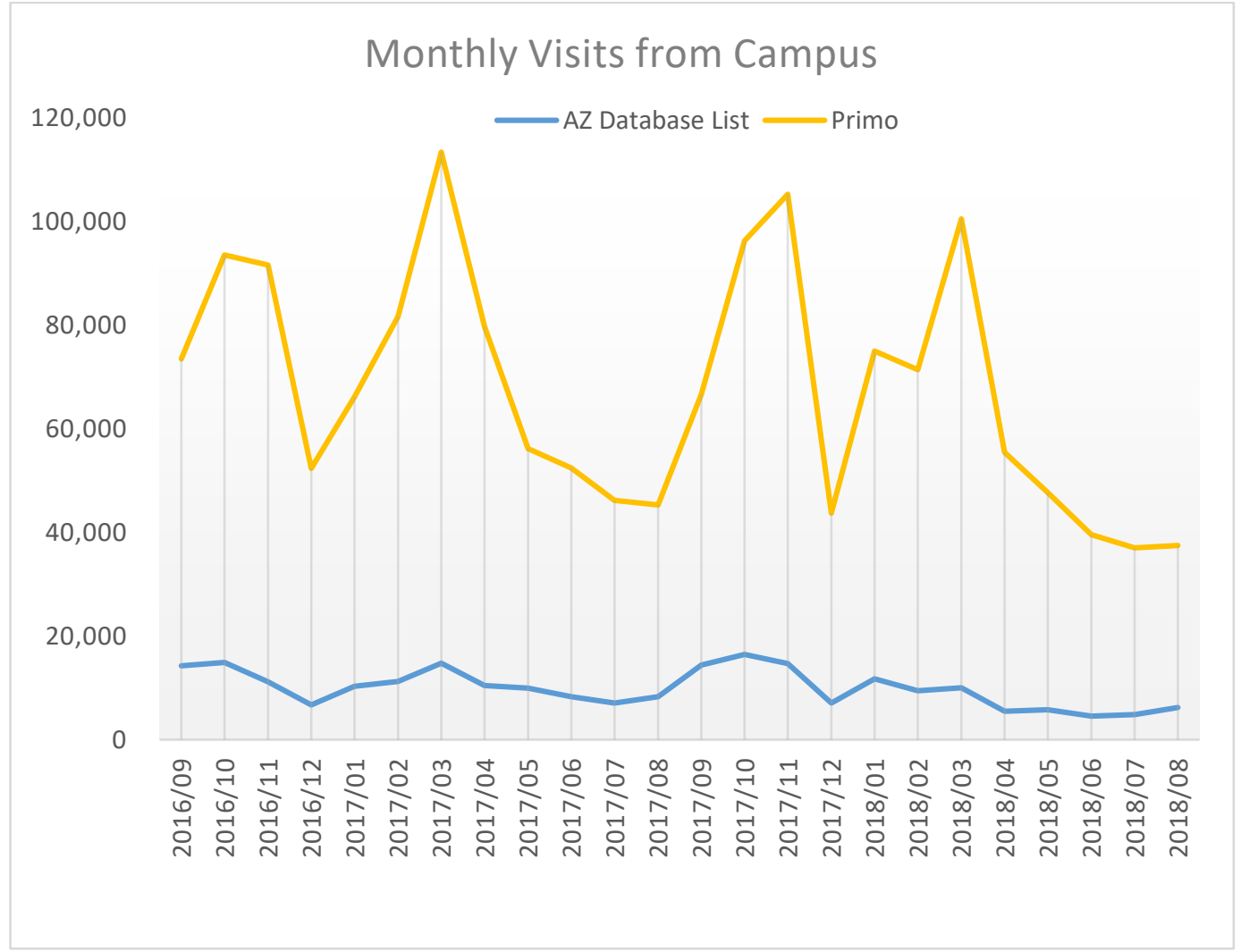

Figure 7. Monthly visits from campus 
Regarding the on-campus visits from the AZ database list, 116,972 visits were made through the University of Manitoba Campus Ethernet. 72,853 visits were made through the WiFi network used by students and guests. The rest 48,029 visits were made through other methods such as some remote libraries on the UM network (Figure 8).

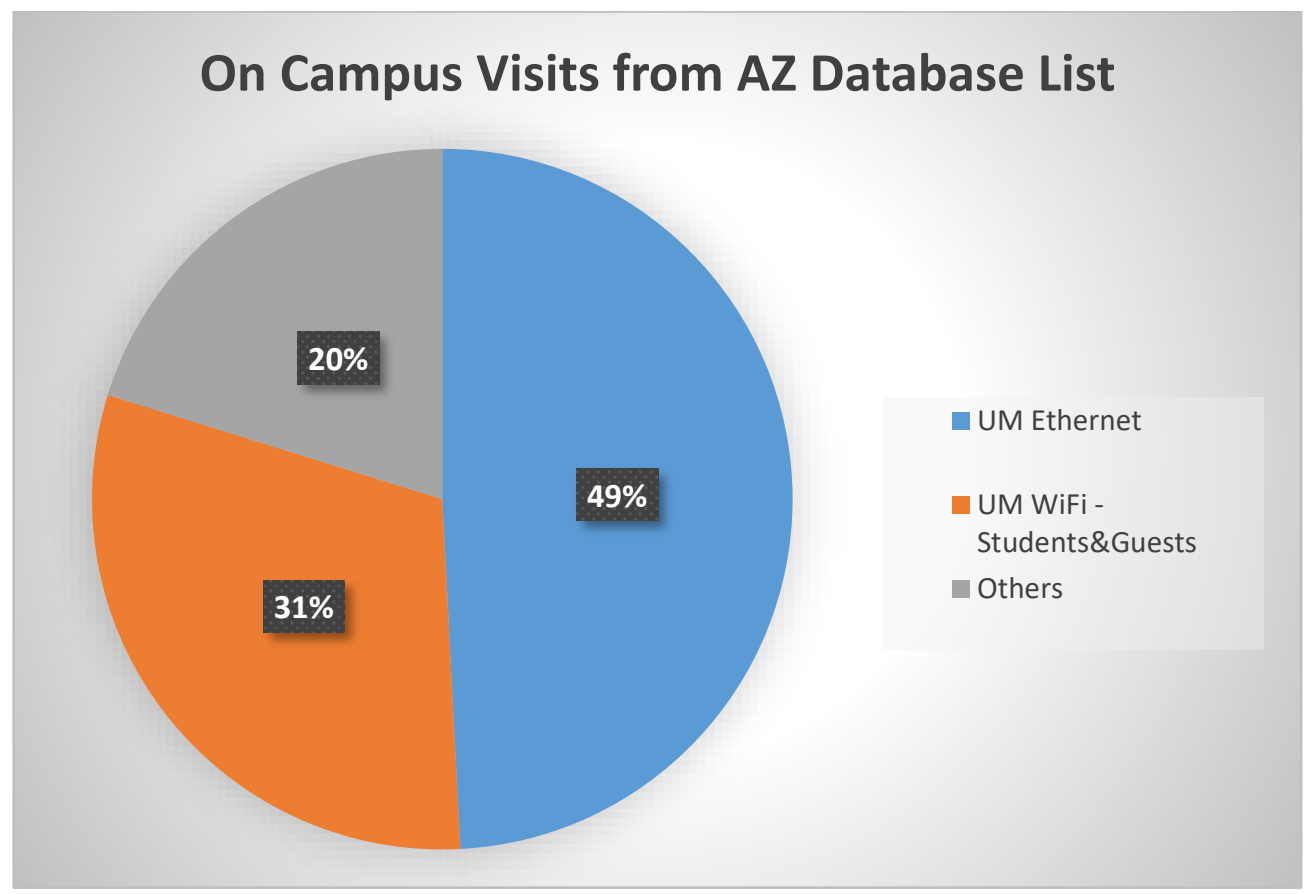

Figure 8. On-campus visits from the AS database list

Regarding the on-campus visits from Primo, 815,864 visits were made through the University of Manitoba Campus Ethernet. 659,817 visits were made through the WiFi network used by students and guests. The rest 151,716 visits were made through other methods such as some remote libraries on the UM network (Figure 9).

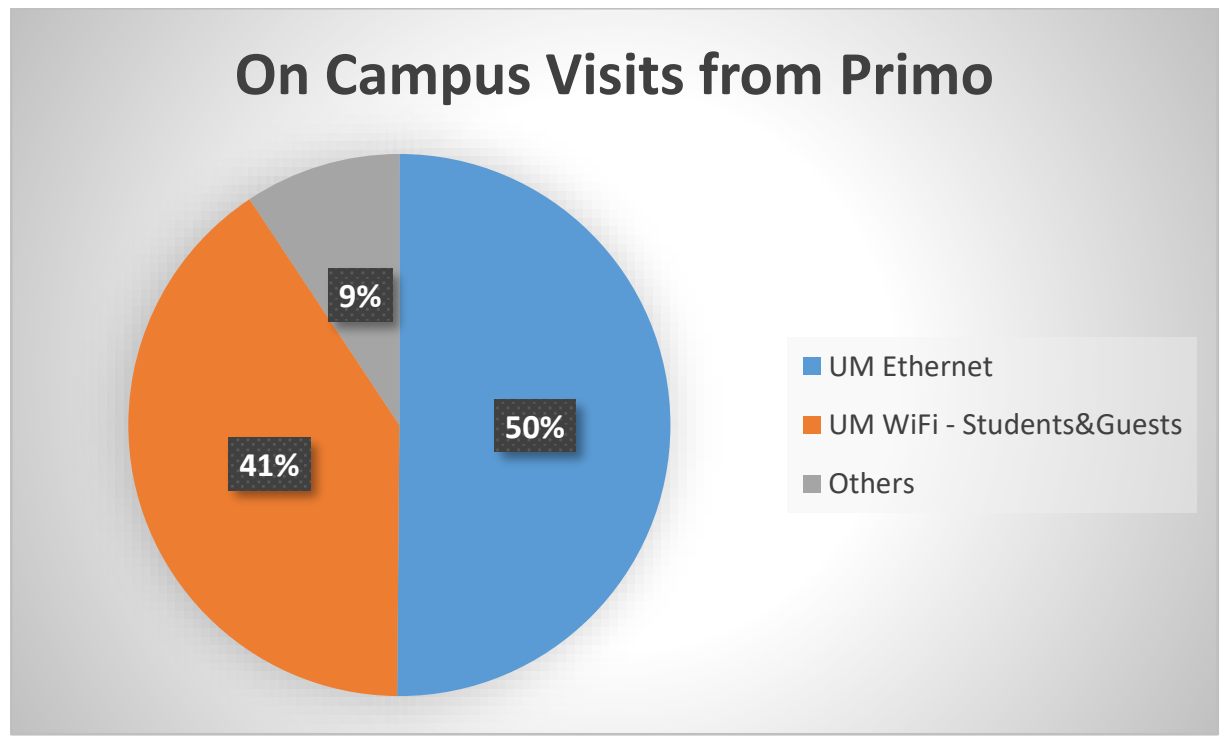

Figure 9. On-campus visits from Primo 
The overall top 10 database domains that were most frequently visited from campus can be found in Table 3. Although dx.doi.org is not a database provider, the importance of digital object identifier (DOI) can be demonstrated by the number of visits to articles with a DOI.

Table 3. Top 10 database domains most frequently visited from campus

\begin{tabular}{|c|c|}
\hline Database Domain & Total Visits \\
\hline www.ncbi.nlm.nih.gov & 116,764 \\
\hline www.sciencedirect.com & 110,951 \\
\hline onlinelibrary.wiley.com & 93,213 \\
\hline search.proquest.com & 90,008 \\
\hline search.ebscohost.com & 87,825 \\
\hline dx.doi.org & 79,923 \\
\hline link.springer.com & 65,473 \\
\hline scholar.google.com & 62,192 \\
\hline www.tandfonline.com & 61,951 \\
\hline openurl.ebscohost.com & 49,189 \\
\hline
\end{tabular}

Regarding the off-campus visits, 606,236 were made from the AZ database list while 2,589,911 were made from Primo (Figure 10. Database visits from off-campus

). The monthly visits from off-campus can be found in Figure 11. Although Primo is still the user's first choice, compared to on-campus usage, more users choose AZ Database List to start their database access.



Figure 10. Database visits from off-campus

The top 10 user groups that made the most database visits from off-campus can be found in Table 4. 


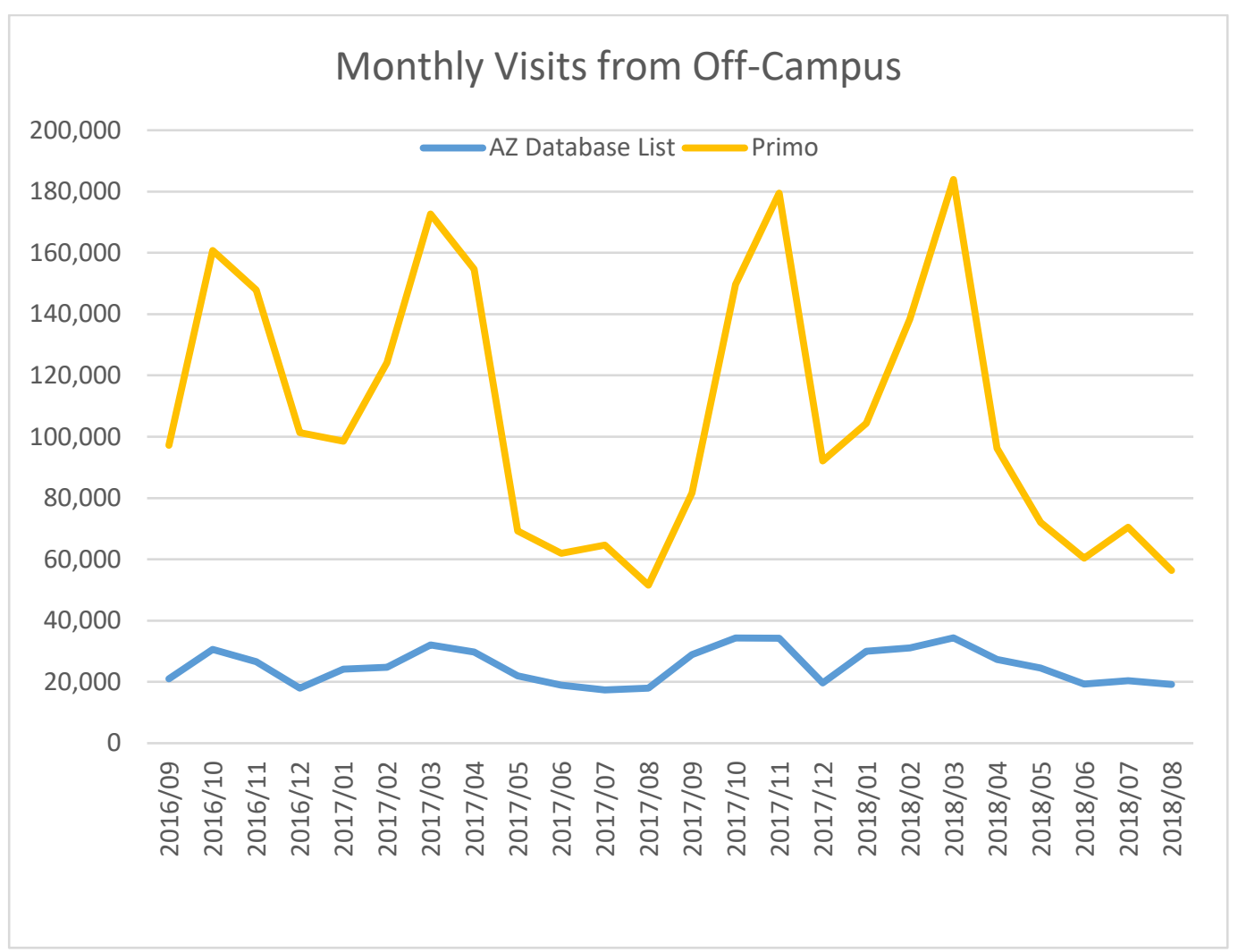

Figure 11. Monthly visits from off-campus

Table 4. Top 10 user groups from off-campus

\begin{tabular}{|c|c|}
\hline User Group & Number Of Visits \\
\hline $\begin{array}{c}\text { Graduate Students } \\
\text { (GCode :16, Faculty of Graduate Studies) }\end{array}$ & 709,249 \\
\hline UofM Faculty and Staff (GCode: 100) & 429,197 \\
\hline $\begin{array}{c}\text { Undergraduate Students from Faculty of Arts } \\
\text { (GCode: } 1 \text { ) }\end{array}$ & 301,185 \\
\hline $\begin{array}{c}\text { Undergraduate Students from Faculty of } \\
\text { Science (GCode: } 2 \text { ) }\end{array}$ & 209,722 \\
\hline $\begin{array}{c}\text { Undergraduate Students from College of } \\
\text { Medicine - PGME (GCode: } 25 \text { ) }\end{array}$ & 204,032 \\
\hline $\begin{array}{c}\text { Undergraduate Students from College of } \\
\text { Medicine (GCode: 5) }\end{array}$ & 113,398 \\
\hline $\begin{array}{c}\text { Undergraduate Students from College of } \\
\text { Pharmacy (GCode: 11) }\end{array}$ & 107,163 \\
\hline $\begin{array}{c}\text { Undergraduate Students from College of } \\
\text { Nursing (GCode: 13) }\end{array}$ & 105,023 \\
\hline $\begin{array}{c}\text { Undergraduate Students from Faculty of } \\
\text { Social Work (GCode: } 12 \text { ) }\end{array}$ & 94,565 \\
\hline
\end{tabular}




\begin{tabular}{|c|c|}
$\begin{array}{c}\text { Undergraduate Students from Faculty of } \\
\text { Education (GCode: } 8 \text { ) }\end{array}$ & 69,215 \\
\hline
\end{tabular}

If we combine all undergraduate students together, they made the most database visits from off-campus. In this study, undergraduate students are affiliated with their respective faculty. Thus, in the current environment, graduate students made the most off-campus visits to the databases within the past two years. The author was interested in what databases were mostly used by these users. The top 10 databases on the AZ database list that were most frequently used by graduate students can be found in Table 5. The top 10 database domains that graduate students visited from Primo can be found in Table 6.

Table 5. Top 10 databases on the AZ database list visited by graduate students from off-campus

\begin{tabular}{|c|c|}
\hline Database Name & Total Visits \\
\hline PubMed & 24,108 \\
\hline UpToDate & 15,669 \\
\hline CINAHL with Full Text & 6,378 \\
\hline Scopus & 5,444 \\
\hline PsycINFO & 4,285 \\
\hline $\begin{array}{c}\text { Web of Science (all } \\
\text { databases) }\end{array}$ & 3,160 \\
\hline RxTx (CPA) & 3,078 \\
\hline MEDLINE (Ovid) & 2,453 \\
\hline LexiComp Online & 2,310 \\
\hline ClinicalKey & 1,677 \\
\hline
\end{tabular}

Table 6. Top 10 database domains visited from Primo by graduate students from off-campous.

\begin{tabular}{|c|c|}
\hline Database Domain & Total Visits \\
\hline www.sciencedirect.com & 48,975 \\
\hline onlinelibrary.wiley.com & 45,381 \\
\hline dx.doi.org & 44,062 \\
\hline www.tandfonline.com & 37,676 \\
\hline scholar.google.com & 36,239 \\
\hline link.springer.com & 35,553 \\
\hline search.proquest.com & 34,534 \\
\hline search.ebscohost.com & 30,024 \\
\hline openurl.ebscohost.com & 29,280 \\
\hline journals.sagepub.com & 24,321 \\
\hline
\end{tabular}

\section{CONCLUSION}

Database usage analysis allows librarians to learn various aspects of how users access the library's e-resources. A simple question like how many times a database is visited within a period is 
fundamental to justify the institution's expenditure. Without the usage analysis, it will be very difficult for a library to investigate a model of the user accessing e-resources based on which custom service could be developed. Compared to the vendor-provided data, transaction $\log$ analysis provides librarians access to first-hand data, allows cross-database comparison, and can be combined with information from other systems for advanced studies. In this article, the author presented a home-grown system used at the University of Manitoba Libraries to analyze EZProxy SPU logs. The author studied how licensed databases were visited within two years (September $1^{\text {st }}, 2019$ - August $31^{\text {st }}, 2019$ ). The author also used transaction log analysis to demonstrate how the database visits performed at different locations and by various user groups change with time.

Since the EZProxy SPU log is being automatically imported into the system daily, it allows the author to analyze the database usage activities within a longer period. Further studies could be focused on the information-seeking behaviors from a particular user group, as such information is available for off-campus visits. This article briefly discussed how users in the Faculty of Graduate Studies group used the e-resources. More studies could be performed in this area to investigate a model of user activities for particular user groups. Meanwhile, although the article demonstrated the roles Ethernet and Wi-Fi play when it comes to database visits from campus, the statistical usage is split into two groups by the fact that IP authentication is used for on-campus visits and ID authentication for off-campus visits. If IP authentication is replaced in the future, on-campus users and off-campus users could be evaluated together and a more comprehensive model could be developed.

\section{Appendix: UserGroup Table}

UserGroup table in the database. Groups 1-15 and 17-29 are used for undergraduate students. Group 16 is for graduate students. UofM faculty and staff are in Group 100.

\begin{tabular}{|c|l|}
\hline GCode & \multicolumn{1}{|c|}{ GroupName } \\
\hline 1 & Faculty of Arts \\
\hline 2 & Faculty of Science \\
\hline 3 & Faculty of Engineering \\
\hline 4 & English Language Centre (2) \\
\hline 5 & College of Medicine \\
\hline 6 & Faculty of Management \\
\hline 7 & Faculty of Agric. and Food Sci. \\
\hline 8 & Faculty of Education \\
\hline 9 & Faculty of Architecture \\
\hline 10 & Faculty of Law \\
\hline 11 & College of Pharmacy \\
\hline 12 & Faculty of Social Work \\
\hline 13 & College of Nursing \\
\hline 15 & School of Art \\
\hline
\end{tabular}




\begin{tabular}{|c|l|}
\hline GCode & \multicolumn{1}{|c|}{ GroupName } \\
\hline 16 & Faculty of Graduate Studies \\
\hline 17 & College of Dentistry \\
\hline 19 & College of Rehabilitation Sci. \\
\hline 20 & School of Dental Hygiene \\
\hline 21 & Faculty of Music \\
\hline 22 & Faculty of Kines. \& Rec Mgmt. \\
\hline 24 & School of Agriculture \\
\hline 25 & College of Medicine - PGME \\
\hline 26 & Extended Education \\
\hline 27 & University 1 \\
\hline 28 & Faculty of Environment, Earth \& Resources \\
\hline 29 & Faculty of Health Sciences \\
\hline 100 & UofM Faculty and Staff \\
\hline 101 & USB Faculty and Staff \\
\hline 200 & Hospital Staff \\
\hline 201 & MRHA \\
\hline 300 & Distance Education \\
\hline
\end{tabular}

\section{References}

Baker, G., \& Read, E. J. (2008). Vendor-supplied usage data for electronic resources: a survey of academic libraries. Learned Publishing, 21(1), 48-57. https://doi.org/10.1087/095315108X247276

Blecic, D., Fiscella, J., \& Wiberley, S. (2001). The measurement of use of Web-based information resources: An early look at vendor-supplied data. College \& Research Libraries, 62(5), 434-453. https://doi.org/10.5860/crl.62.5.434

Blecic, D., Fiscella, J., \& Wiberley, S. (2007). Measurement of Use of Electronic Resources: Advances in Use Statistics and Innovations in Resource Functionality. College \& Research Libraries, 68(1), 26-44. https://doi.org/10.5860/crl.68.1.26

Coombs, K. A. (2005). Lessons learned from analyzing library database usage data. Library Hi Tech, 23(4), 598-609. https://doi.org/10.1108/07378830510636373

COUNTER. (2019). What is COUNTER? Retrieved July 25, 2019, from https://www.projectcounter.org/about/

Duy, J., \& Vaughan, L. (2003). Usage data for electronic resources: A comparison between locally collected and vendor-provided statistics. Journal of Academic Librarianship, 29(1), 16-22. https://doi.org/10.1016/S0099-1333(02)00400-7

Gonzales, B. M. (2018). Analyzing EZproxy SPU Logs Using Python Data Analysis Tools. Code4Lib Journal, (42).

Liu, G., \& Fu, P. (2018). Shared Next Generation ILSs and Academic Library Consortia: Trends, Opportunities and Challenges. International Journal of Librarianship (IJoL), 3(2), 53-71. https://doi.org/10.23974/ijol.2018.vol3.2.94

NISO. (2019). SUSHI History/Origins. Retrieved July 25, 2019, from 
https://www.niso.org/standards-committees/sushi/historyorigins

OCLC. (2018a). LogSPU. Retrieved from

https://help.oclc.org/Library_Management/EZproxy/Configure_resources/LogSPU

OCLC. (2018b). Starting point URLs and config.txt.

OCLC. (2019). Log files overview. Retrieved July 24, 2019, from https://help.oclc.org/Library_Management/EZproxy/Manage_EZproxy/Log_files_overview

OED, O. (2019). database, n. Retrieved from www.oed.com/view/Entry/47411

Peters, T. A. (1993). The history and development of transaction log analysis. Library Hi Tech, 11(2), 41-66. https://doi.org/10.1108/eb047884

Shibboleth. (2019). Shibboleth. Retrieved July 25, 2019, from https://www.shibboleth.net

Wan, G., \& Liu, Z. (2010). Knowing your users: The value of article database usage analysis. Learned Publishing, 23(3), 225-236. https://doi.org/10.1087/20100305

\footnotetext{
About the author

Wei Xuan is Assistant Librarian and Head of Libraries Systems at the University of Manitoba Libraries. He has been playing an active role in numerous projects focused on systems interoperability, data protection and accessibility. His current research interests include resource evaluation, data management and library technology.
} 